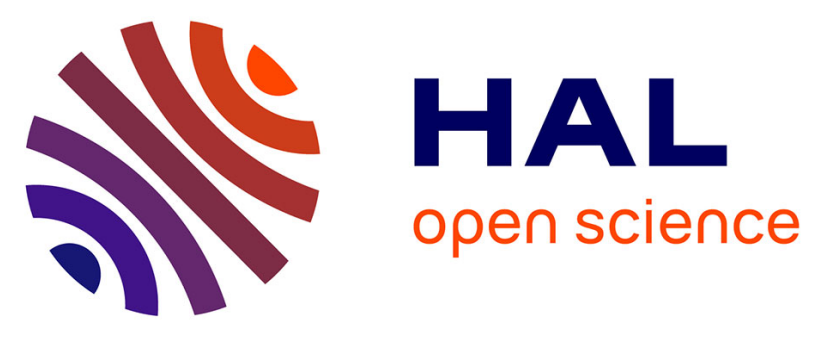

\title{
Physical interaction between the histone acetyl transferase Tip60 and the DNA double strand breaks sensor MRN complex
}

Catherine Chailleux, Sandrine Tyteca, Christophe Papin, François Boudsocq, Nadine Puget, Céline Courilleau, Mikhaïl Grigoriev, Yvan Canitrot, Didier Trouche

\section{To cite this version:}

Catherine Chailleux, Sandrine Tyteca, Christophe Papin, François Boudsocq, Nadine Puget, et al.. Physical interaction between the histone acetyl transferase Tip60 and the DNA double strand breaks sensor MRN complex. Biochemical Journal, 2010, 426 (3), pp.365-371. 10.1042/BJ20091329 . hal00479248

\section{HAL Id: hal-00479248 \\ https://hal.science/hal-00479248}

Submitted on 30 Apr 2010

HAL is a multi-disciplinary open access archive for the deposit and dissemination of scientific research documents, whether they are published or not. The documents may come from teaching and research institutions in France or abroad, or from public or private research centers.
L'archive ouverte pluridisciplinaire HAL, est destinée au dépôt et à la diffusion de documents scientifiques de niveau recherche, publiés ou non, émanant des établissements d'enseignement et de recherche français ou étrangers, des laboratoires publics ou privés. 
Physical interaction between the histone acetyl transferase Tip60 and the DNA double strand breaks sensor MRN complex

Catherine Chailleux* $\S$, Sandrine Tyteca* $\S$, Christophe Papin $†$, François Boudsocq $†$, Nadine Puget $\ddagger$, Céline Courilleau*, Mikhaïl Grigoriev†, Yvan Canitrot*, Didier Trouche*

From LBCMCP*, LBME†, and IPBSł, CNRS and University of Toulouse, 31062 Toulouse, France

Corresponding author: Didier Trouche, LBCMCP UMR 5088 CNRS/UPS, 118 Route de Narbonne, 31062 Toulouse Cedex 9. Phone : +33 561558579 ; Fax : +33 561558109 ; E-mail : $\underline{\text { dtrouche@cict.fr }}$

Short title: "physical interaction between Tip60 and the MRN complex"

$\S$ These authors contributed equally to this work. 


\section{Synopsis}

Chromatin modifications and chromatin modifying enzymes are believed to play a major role in the process of DNA repair. The Tip60 histone acetyl transferase is physically recruited to DNA double strand breaks (DSB) where it mediates histone acetylation. Here, we show using a reporter system in mammalian cells that Tip60 expression is required for homology-driven repair, strongly suggesting that Tip60 participates in DNA DSB repair through Homologous Recombination. Moreover, Tip60 depletion inhibits the formation of Rad50 foci following ionizing radiations, indicating that Tip60 expression is necessary for the recruitment of the DNA damage sensor MRN (Mre11, Rad50, Nbs1) complex to DNA DSB. Moreover, we found that endogenous Tip60 physically interacts with endogenous MRN proteins in a complex which is distinct from the classical Tip60 complex. Taken together, our results describe a physical link between a DNA damage sensor and a histone modifying enzyme and provide important new insights into the role and mechanism of action of Tip60 in the process of DNA DSB repair.

Keywords

Tip60, histone acetylation, chromatin, DNA repair, MRN complex

Abbreviations : DSB : double strand breaks; HDR : homology-directed repair; HR : Homologous Recombination;IR : ionizing radiation; MRN : Mre11-Rad50-Nbs1; NHEJ : Non-Homologous End Joining. 


\section{Introduction}

All processes requiring access to DNA have to deal with DNA compaction in chromatin. Chromatin modifications and chromatin modifying machineries have been extensively studied in transcriptional control. However, it is now clear that they are also critical in other processes such as DNA repair. In particular, DNA double strand breaks (DSB) are extremely deleterious damages, which require rapid and efficient repair. Chromatin modifications are known to be important for signaling and dynamic of the DNA DSB repair machineries. In yeast, chromatin remodeling following DNA DSB has been extensively studied and the machineries involved characterized [1].

In mammals, although far less is known, the involvement of the histone acetyl transferase Tip60 has been clearly demonstrated [2]. Tip60 belongs to the MYST family of histone acetyl transferases, which is conserved from yeast to human [3]. In agreement with its role on histone acetylation, Tip60 has been widely described as a transcriptional co-activator. Its expression is required for myc and p53-dependent genes transcription [4, 5]. Tip60 belongs to a multimolecular complex, called the Tip60 complex [6]. The Tip60 complex is considered as the human orthologue of two yeast complexes, the NuA4 histone acetyl transferase complex and the SWR1 chromatin remodeling complex, both known to be involved in DNA DSB repair [7]. In mammals, a protein belonging to the Tip60 complex, TRRAP, has been shown to be important for both Homologous Recombination (HR) and Non-Homologous End Joining (NHEJ), the two main pathways of DNA DSB repair [2, 8]. Tip60 itself is recruited around DNA DSB, where it mediates histone acetylation and subsequent recruitment to repair sites of DNA repair factors, such as Rad51 [2]. In addition to its role in local histone acetylation, Tip60 activity is important for DNA DSB signaling: following DNA DSB, it can acetylate the transducer kinase ATM, this acetylation being critical for ATM autophosphorylation and activation $[9,10]$.

Here we investigate the role of the Tip60 complex in DNA DSB repair. We identify a new multimolecular complex containing Tip60 and the MRN complex, a sensor of DNA DSB. Altogether, our results strongly suggest that this Tip60/MRN complex is important for the repair of DSB by HR.

\section{Experimental}

\section{Cell culture}

Jurkat cells were cultured in RPMI supplemented with 10\% FCS. All other cells were cultured in DMEM supplemented with 10\% FCS. The HeLa cell line stably overexpressing HA-Tip60 was kindly provided by V. Ogryzko. The U2OS cell line containing an integrated reporter substrate to measure Homology-Directed Repair of a single DSB has been previously described [11]. Irradiation was performed using a Xray Faxytron device. Calicheamicin was provided by Wyeth. HeLa Nuclear Extracts were purchased from Computer Cell Culture Center (Belgium).

\section{Antibodies}

The polyclonal anti-HA Ab (Y-11) was purchased from Santa Cruz. The anti-Rad50 (13B3), anti-Nbs1 (1C3) and anti-Mre11 (12D7) Abs were purchased from GeneTex. The antiphosphoATM (S1981), $\gamma$-H2AX and anti-HDAC3 Abs were purchased from Cell Signaling Technologies. The anti-phosphoNbs1 (S343) was purchased from Interchim. We used two anti-ATM Ab (AB-3, Calbiochem; ab17995, Abcam). The anti-p400 antibody was purchased from Abcam. The anti-Tag myc Ab (9E10) was purchased from Roche Diagnostics. The antiTTRAP antibody (2TRR1B3) was a kind gift from Dr L. Tora. The anti-Tip60 DT antibody was previously described [12]. The anti-Tip60 CLHF; RLPV and CLGT antibodies were king gifts from Dr B. Amati and are described in [4]. The anti-Tip60LM antibody was raised and 
purified in DT's laboratory using the CLGT and CLHF peptides described in [4]. Secondary Abs were purchased from Amersham (peroxydase-conjugated) or Molecular Probes (dyeconjugated). Details on the Abs dilutions used are available upon request.

\section{Plasmids, siRNAs and transfection methods}

All siRNAs were purchased at Eurogentec. The C1, C2 and Tip60 siRNAs are described in [5]. The sequence of the top strands of the Rad50, Mre11 and TRRAP siRNAs were respectively GCUAAUGACUCUGAUGAUA-dTdT, GGAGAAGGAAAUACCAGAAdTdT and GAAGUGUAAGCCUCAGUCA- dTdT. siRNAs were transfected by electroporating $5.10^{6}$ cells in $200 \mu \mathrm{L}$ of serum-free OptiMEM with $15 \mu \mathrm{L}$ siRNA $(100 \mu \mathrm{M})$ using a BioRad electroporation device set to $250 \mathrm{~V}, 950 \mu \mathrm{F}$ or using an Amaxa device according to the manufacturer's instructions. For transfection and expression of the I-SceI endonuclease, cells were transfected by calcium phosphate co-precipitation with the pcDNA3ßmycNLS-I-SceI plasmid. As a control for the transfection efficiency, a GFPencoding plasmid was used. The plasmid expressing untagged Tip60 (pcDNA3 Tip60, details of construction are available upon request) or empty vector (pcDNA3) were transfected by calcium phosphate co-precipitation.

\section{Clonogenic assay}

U2OS cells electroporated with the various siRNAs were plated in T25 flasks at a density of $10^{3}$ or $2.10^{3}$ cells per flask, then irradiated. For Mitomycin C (Sigma) cytotoxicity cells were treated with increasing concentrations of drug for $1 \mathrm{~h}$. Ten days later, cells were stained with crystal violet and colonies containing more than 50 cells were counted.

\section{HR assay}

Cells were seeded at $2 \times 10^{5}$ in 6 -well plates. Recombination was induced by transient transfection of cells with $2 \mu \mathrm{g}$ of the pcDNA $3 \beta-N L S-I-S c e I$ plasmid. Transfection efficiency was measured by the cotransfection of a mixture of GFP plasmid $(0.2 \mu \mathrm{g})$ with the pBlueScript plasmid $(1.8 \mu \mathrm{g})$ to obtain equivalent amount of transfected DNA. Three days after transfection cells were washed with PBS and trypsinized. Cells were resuspended in PBS and recombination was measured by the quantification of GFP positive cells by flow cytometry (FACScalibur, Becton Dickinson). Quantification was performed on $2.5 \times 10^{4}$ sorted events. Relative fraction of DSB-induced Homologous Recombination was normalized to the transfection efficiency obtained for each cell treatment condition.

\section{Preparation of nuclear extracts, immunoprecipitations and immunodepletions}

Preparation of Jurkat nuclear extracts, immunoprecipitations and immunodepletions were performed as described previously [13]. Transfected U2OS cells were harvested using trypsin, resuspended in five cell pellets volumes of lysis buffer $(10 \mathrm{mM}$ Tris $\mathrm{pH} 8.0,10 \mathrm{mM}$ $\mathrm{NaCl}, 2 \mathrm{mM} \mathrm{MgCl} 2$ supplemented with protease inhibitors). After a 5 min incubation on ice, NP40 was added $(0.5 \%$ final $)$ and cells were incubated on ice for $5 \mathrm{~min}$. After centrifugation at $2000 \mathrm{~g}$ for $5 \mathrm{~min}$, supernatant was discarded, and pellet was resuspended in buffer 3 (equal volume to the cell pellet) (20mM Hepes pH 7.9, $420 \mathrm{mM} \mathrm{NaCl}, 1.5 \mathrm{mM} \mathrm{MgCl} 2,0.2 \mathrm{mM}$ EDTA). After a $30 \mathrm{~min}$ incubation at $4^{\circ} \mathrm{C}$, samples were centrifugated at $20000 \mathrm{~g}$ and supernatants (nuclear extracts) were collected.

\section{Western Blotting}

For Western Blot analysis, samples were separated either by SDS-PAGE or on a NuPAGE® novex 3-8\% Tris-acetate gel (Invitrogen). Proteins were then transferred respectively to nitrocellulose membrane or to PVDF membrane. Specific primary antibodies as well as peroxidase-conjugated secondary antibodies were used according to standard western blot 
procedures and peroxidase activity was then detected by using LumiLight Plus reagent (Roche).

\section{Immunofluorescence}

Cells seeded on glass coverslips were fixed with formaldehyde (3.7\%) for $20 \mathrm{~min}$ at RT then permeabilized with Triton X100 (0.1\%) for $5 \mathrm{~min}$ at RT. Coverslips were mounted with the Vectashield antifade solution containing DAPI (Molecular probes). Images were collected using a digital camera attached to a Leica (DM5000) fluorescence microscope. Digital images were prepared for publication using Metamorph software.

\section{Size Exclusion Chromatography}

Nuclear extract ( $2 \mathrm{ml}, 3 \mathrm{mg}$ ) was clarified by a centrifugation at $10000 \mathrm{rpm}$ and was loaded on a 10/300 GL Superdex 200 column (GE Healthcare) preequilibrated in $120 \mathrm{~mL}$ of Buffer (20ml Tris pH7.5, 100mM KCl, 10\% Glycerol, 1mM DTT). The column was run at a flow rate of $0.2 \mathrm{~mL} / \mathrm{min}$, and $1.5 \mathrm{~mL}$ fractions were analyzed for absorbance at $280 \mathrm{~nm}$ and for the presence of Tip60, p400, MRN by Western blot.

\section{Results}

\section{Tip60 is required for DSB repair by HR}

To test whether Tip60 is important for DSB repair, we transfected U2OS cells with a siRNA directed against Tip60 (described in [5]). This siRNA specifically decreased the expression of Tip60 (Fig. 1A). We next performed a clonogenic assay after irradiation (IR). We found that cells transfected by the Tip60 siRNA were more sensitive to IR than cells transfected by the control siRNA, indicating that Tip60 influences DSB repair (Fig. 1A). Cells defective in the Homologous Recombination (HR) pathway are known to display increased sensitivity to IR [14]. To examine a potential defect in the HR pathway we treated the cells with mitomycin C since sensitivity to this drug is a hallmark of a defective HR pathway [15]. Again, we found that transfection of a siRNA against Tip60 leads to increase sensitivity to mitomycin C (Fig. 1A). Altogether, these drug sensitivity studies suggest that Tip60 is important for HR. To confirm this finding directly, we used U2OS cells containing a reporter system (described in supplementary data 1) for homology-directed repair (HDR) [11], a process highly related to HR. The HDR of a unique DNA DSB induced at an I-SceI site on a transgene leads to the appearance of GFP-positive cells. As positive controls, we transfected U2OS cells with siRNAs against two components of the MRN complex, Rad 50 or Mre11 (see supplementary data 2 for western blots demonstrating the efficiency of Rad50 and Mre11 siRNA). As previously described [16], we observed a decrease of HDR after transfection with the Mre11 siRNA [16] as well as with the Rad50 siRNA (compared to the control siRNA) (see supplementary data 3 for representative FACS data for each sample), therefore demonstrating that our assay allows to assess adequately for the involvement of proteins in the HR pathway. Strikingly, transfection of two Tip60 siRNAs also significantly decreased the number of GFP-positive cells (Fig. 1B). Since the expression of I-SceI was not affected by Tip60 knockdown (Fig. 1B), this result indicates that Tip60 expression is required for HDR.

Tip60 expression is required for induction of $\gamma \mathrm{H} 2 \mathrm{AX}$ and $\mathrm{Rad50}$ foci in U2OS cells We then intended to characterize the molecular event controlled by Tip60. We first investigated the role of Tip60 in phosphorylated $\mathrm{H} 2 \mathrm{AX}(\gamma \mathrm{H} 2 \mathrm{AX})$ foci formation in U2OS cells. Indeed, conflicting results have been previously reported since two studies found that depleting Tip60 leads to increased $\gamma \mathrm{H} 2 \mathrm{AX}$ levels $[17,18]$, whereas two others describe the opposite $[19,20]$. We thus transfected U2OS cells by a Tip60 siRNA, we irradiated cells for 1 and 6 hours and we performed a $\gamma \mathrm{H} 2 \mathrm{AX}$ immunofluorescence. Irradiation induces the appearance of a strong punctuated $\gamma \mathrm{H} 2 \mathrm{AX}$ staining on most of the cells (Fig. 2A, see 
supplemental data 4 for enlargement of typical cells). To quantify precisely and unequivocally the effect of the Tip60 siRNA, we applied a threshold on $\gamma \mathrm{H} 2 \mathrm{AX}$ staining using the Image J software and calculated the percentage of cells which stained positive for $\gamma \mathrm{H} 2 \mathrm{AX}$ (on 250 independent cells). We found that transfection of the Tip60 siRNA decreases the amount of $\gamma \mathrm{H} 2 \mathrm{AX}$-positive cells (Fig. 2B). Moreover, the effects of Tip60 on $\gamma \mathrm{H} 2 \mathrm{AX}$ induction were also observed when the total $\gamma \mathrm{H} 2 \mathrm{AX}$ fluorescence levels per cell were averaged on 500 independent cells (supplementary data 5). Tip60 knockdown inhibited $\gamma \mathrm{H} 2 \mathrm{AX}$ induction as soon as 1 hour following irradiation, indicating that Tip60 expression is required for efficient phosphorylation of $\mathrm{H} 2 \mathrm{AX}$ in U2OS cells. We then tested the effects of Tip60 knockdown on one of the first events observed following DSB induction, which is the recruitment of the MRN complex to DSB. MRN recruitment to damaged DNA can be visualized by the appearance of foci following induction of DNA DSB [21]. We thus transfected U2OS cells with the Tip60 siRNA, irradiated them and stained them with a Rad50 antibody (Fig. 3A, see supplementary data 4 for enlargement of a typical cell). We performed the quantification of foci formation by blind counting of the percentage of foci-presenting cells from 250 independent cells (although most cells presented multiple foci (see Fig. 3A), cells were recorded as positive as soon as they had one foci). Quantification is shown in Fig. 3B. We found that six hours following irradiation, about $80 \%$ of control cells harbored Rad50 foci in cells transfected by the control siRNA (Fig. 3A and B,). In cells transfected by the Tip60 siRNA, the number of cells presenting foci was significantly reduced. Again, this effect was also observed in an early time point (1 hour), indicating that Tip60 controls the formation of MRN foci rather than their maintenance. Since Tip60 down-regulation did not significantly affect Rad50 expression, nor the expression of other members of the MRN complex (Fig. 3C), this result indicates that Tip60 expression is important for the efficient recruitment of Rad50 and likely of the MRN complex to damaged DNA.

\section{Endogenous Tip60 and MRN proteins physically interact}

Interestingly, a molecular link between Tip60 and the MRN complex was recently described, since MRN proteins interact with TRRAP, a component of the Tip60 complex [8], suggesting that the effects described above could be due to a physical interaction between Tip60 and MRN. To test this possibility, we immunoprecipitated endogenous Tip60 from HeLa nuclear extracts and we tested the presence of endogenous MRN proteins and TRRAP in the immunoprecipitates by western blot (Fig. 4A). As expected, immunoprecipitation of Tip60 led to the co-immunoprecipitation of TRRAP protein. Moreover, we found that all three MRN proteins were detected in the Tip60 IP and not the control IP (Fig. 4A). Detection of endogenous Tip60 in the MRN immunoprecipitates was impossible since endogenous Tip60 co-migrates with the immunoglobulin heavy chains (data not shown). Thus, to rule out the possibility that the presence of MRN proteins in the Tip60 IP shown in Figure 4A was due to a cross-reaction of the Tip60 antibody with another protein, we performed immunoprecipitation of endogenous Tip60 with three other unrelated anti-Tip60 antibodies (kind gift from Dr B. Amati). We detected endogenous MRN proteins in all four immunoprecipitates (Fig. 4B). Taken together, these data indicate that Tip60 and the MRN complex interact at endogenous levels.

Note that Robert et al. failed to detect such a complex between Tip60 and MRN proteins following IP of Nbs1 or Mre11 proteins [8], perhaps because the presence of Tip60 masks the antibodies epitopes. Alternatively, since Robert et al tested the presence of Tip60 using a HAT assay, Tip60 bound by MRN may not be able to acetylate histones (see discussion). Also, MRN proteins were not found associated with overexpressed tagged Tip60 [6]. We did not detect any MRN protein co-immunoprecipitating with Tip60 from the cell line expressing the tagged Tip60 previously used to purify the Tip60 complex (supplementary data 6), strongly suggesting that the addition of a tag at the N-terminus of Tip60 is detrimental for its 
association with MRN proteins.

\section{MRN does not interact with the classical Tip60 complex}

The next question we addressed was whether the whole Tip60 complex associates with MRN proteins. To this aim, we immunodepleted endogenous p400 using an antibody directed against p400, a component of the classical Tip60 complex [22], or with an anti-HA antibody as a control. Endogenous p400 protein was efficiently immunodepleted from Jurkat nuclear extracts (Fig. 5A). Moreover, immunodepletion of p400 led to the depletion of the endogenous Tip60 complex, since Tip60 was also depleted by p400 immunodepletion (Fig. 5A). As expected, the amount of p400 immunoprecipitated by Tip60 were decreased upon p400 immunodepletion (Fig. 5B), confirming that the classical Tip60 complex was indeed depleted. In contrast, the amount of endogenous Rad50 and Nbs1 proteins in the Tip60 IP was not decreased (Fig. 5C), suggesting that the Tip60 protein which interacts with MRN proteins is not physically associated with $\mathrm{p} 400$.

To confirm this finding, we prepared Jurkat nuclear extracts and fractionated them by size exclusion chromatography. By western blot experiments, we found that $\mathrm{p} 400$ was eluted in fractions 5, 6 and 7 whereas the MRN proteins were observed in fractions 5 to 9 (Fig. 5D). A band migrating at $60 \mathrm{kDa}$ is observed in fractions 5 to 11 , probably corresponding to endogenous Tip60. Thus, this experiment suggests that MRN and Tip60 are both present in fractions in which there is no detectable p400 (fractions 8 and 9). To test whether they are physically associated in these fractions, we performed immunoprecipitation experiments using fractions 5 and 6 (which contain Tip60, MRN and p400) and 8 and 9 (which contain MRN and Tip60 but not p400). Immunoprecipitation of Tip60 led to the coimmunoprecipitation of p400 from fractions 5 and 6 and of Rad50 from fractions 5, 6 and 8 (Fig. 5E). This result thus indicates that the classical Tip60 complex elutes in fraction 5 and 6 whereas the Tip60/MRN complex elutes in fraction 5, 6 and 8. Importantly, since p400 is not detected in direct western blots from fraction 8 or in Tip60 IP from fraction 8 , this result indicates that the Tip60/MRN complex can be devoid of p400. Taken together, Fig. 5 results thus indicate that the MRN-associated Tip 60 protein is not embedded within the classical p400-containing Tip60 complex.

We next investigated how Tip60 contacts the MRN complex. We could not detect any interaction between Tip60 and MRN proteins in GST-pull down experiments (data not shown) suggesting that the interaction is not direct. Since TRRAP is known to be physically associated with Tip60 on one hand [6] and with the MRN complex on the other hand [8], we tested whether TRRAP mediates the Tip60/MRN interaction. We transfected U2OS cells with a siRNA directed against TRRAP or with a control siRNA and with an untagged Tip60 expression vector or the corresponding empty vector. As expected TRRAP expression was decreased in cells transfected by the TRRAP siRNAs (Fig. 6). We then prepared nuclear extracts and immunoprecipitated these extracts with a Tip60 antibody (or without antibody as a control). In cells transfected by the control siRNA, overexpression of untagged Tip60 led to a large increase in the amount of p400 and Rad50 co-immunoprecipitating with Tip60 (Fig. 6), respectively reflecting formation of the classical Tip60 complex and of the Tip60/MRN complex. Depletion of TRRAP does not affect the amount of p400 co-immunoprecipitating with Tip60 (Fig, 6), indicating that TRRAP expression is not required for the Tip60/p400 interaction. However, the amount of Rad50 co-immunoprecipitating with Tip60 was strongly decreased upon TRRAP knock-down although TRRAP siRNA had no effect on Rad50 expression. Thus, these data indicate that TRRAP expression is required for the Tip60/Rad50 interaction, strongly suggesting that TRRAP physically bridges Tip60 and MRN proteins through direct or indirect interactions.

\section{Discussion}


In conclusion, we show here that Tip60 expression is required for a very early step of DNA double strand breaks repair, since Tip60 depletion affects the earliest known molecular events occurring following DSBs induction, which are $\gamma \mathrm{H} 2 \mathrm{AX}$ foci formation and MRN complex recruitment. In addition, this role of Tip60 is likely to be direct since we demonstrate that endogenous Tip60 and the sensor MRN complex belongs to the same multimolecular complex (named "the Tip60/MRN complex" below). We thus uncover a physical interaction between a sensor complex and a chromatin modifying enzyme. We further characterize this Tip60/MRN complex by demonstrating that 1) it does not involve the whole Tip60 complex; 2 ) it requires endogenous TRRAP expression strongly suggesting that TRRAP physically bridges Tip60 and the MRN complex. It is important to note that the Tip60/MRN complex likely contains other proteins, since it elutes from size exclusion chromatography experiments as a complex of about $1 \mathrm{MDa}$. These uncharacterized proteins may participate, together with TRRAP, in structuring the Tip60/MRN complex.

The interaction between Tip60 and MRN proteins requires TRRAP. As such, we provide insights into the mechanism by which TRRAP participates in DNA repair. Interestingly, TRRAP regulates the transcription of Mad1 and Mad2 genes through the recruitment of Tip60, which acetylates H4, and GCN5, which acetylates H3 [23]. It would thus be important to test whether TRRAP can also target the GCN5 acetyl transferase to MRN proteins, and thus potentially to DNA DSB. In addition, we demonstrate that the Tip60/MRN complex is different from the classical complex. Interestingly, our overexpression experiments suggest that Tip60 expression is rate limiting for the formation of these two complexes, since we immunoprecipitate more $\mathrm{p} 400$ and Rad50 in the presence of exogenous Tip60. This suggests that the two Tip60-containing complexes could be competing for limiting amounts of Tip60. This possibility underlines the importance of understanding what regulates the presence of Tip60 in one complex or the other. Moreover, disturbing the formation of one complex could indirectly affect the function of the other complex.

What could be the role of the Tip60/MRN complex we uncover here? Clearly, it is likely to play a major role in DNA DSB repair since the five characterized components of this complex are known to favour homologous recombination (this study for Tip60 and Rad50, [16] for Mre11, [2] for TRRAP, [24] for Nbs1). Interestingly, we found that Tip60 expression is required for efficient formation of Rad50 foci. It is tempting to speculate that the Tip60/MRN complex we characterized here is important for Rad50 foci formation. One could imagine for example, that the physical interaction with Tip60 helps the recruitment of MRN proteins to DSB. Alternatively, since Tip60 is an enzyme, it can acetylate a MRN protein in the context of the Tip60/MRN complex therefore favouring its recognition of DNA DSB. In this regard, it is important to note that Nbs1 was recently shown to be acetylated [25].

In addition, since both Tip60 and MRN proteins are known to be required for ATM activation [26], the Tip60/MRN complex could participate in local ATM activation. In agreement with such an hypothesis, we recently showed that ATM activation following oncogenic stress requires Tip60 but not p400 [27], suggesting that this role of Tip60 is not mediated within the classical Tip60 complex. Along this line, it is tempting to speculate that the Tip60/MRN complex is the enzymatic complex which mediates ATM acetylation [9]. Strikingly, Tip60 is involved in two different steps upon DNA DSB, each of these steps being characterized by acetylation of a specific substrate. Through acetylation of ATM, Tip60 is involved in DNA damage signaling [9] and through nucleosome acetylation, it is required for repair proteins recruitment [2] or removal of $\mathrm{H} 2 \mathrm{AX}[17,28]$. These two distinct acetylation events could be mediated by two distinct enzymatic complexes, with the Tip60/MRN complex being the actual enzyme acetylating ATM and the classical Tip60 complex being involved in histone acetylation, since only this latter complex can acetylate nucleosomes [6]. If this hypothesis is true, then Tip60 would be highly dynamic during DSB repair. First, it would be recruited 
very rapidly with MRN proteins to allow proper signaling through ATM acetylation. Then the classical Tip60 complex would be recruited to mediate histone acetylation. Interestingly, such a model is supported by our recent findings that knock-down of $\mathrm{p} 400$ favours the activation of Tip60-dependent DNA damage pathways [27]. One could imagine that in cells in which p400 levels are decreased, Tip60 is redistributed to the Tip60/MRN complex therefore allowing a stronger response to DNA damage. Studies of the recruitment dynamic and interdependence of the various Tip60-containing complexes are required to validate such a model.

\section{Acknowledgments}

The authors wish to thank Drs B. Amati, V. Ogryzko, L. Tora and B.S. Lopez for materials, Dr B.S. Lopez for critical reading of the manuscript, C. Lorenzo for assistance with fluorescence microscopy and M. Quaranta for assistance with flow cytometry. We acknowledge the use of the imaging facilities of CBD - IFR109 of Toulouse.

\section{Funding}

This work was supported by a grant from the ARC as a "subvention libre", from the ANR [ANR-06-3-13-7148], from the "Ligue Nationale Contre le Cancer" as an "équipe labellisée" and from "Electricité De France". ST was the recipient of a student ship from the ARC.

\section{Numbered references}

1 Downs, J. A. and Cote, J. (2005) Dynamics of chromatin during the repair of DNA double-strand breaks. Cell Cycle. 4, 1373-1376.

2 Murr, R., Loizou, J. I., Yang, Y. G., Cuenin, C., Li, H., Wang, Z. Q. and Herceg, Z. (2006) Histone acetylation by Trrap-Tip60 modulates loading of repair proteins and repair of DNA double-strand breaks. Nat. Cell. Biol. 8, 91-99.

3 Utley, R. T. and Cote, J. (2003) The MYST family of histone acetyltransferases. Curr. Op. Microbiol. Immunol. 274, 203-236.

4 Frank, S. R., Parisi, T., Taubert, S., Fernandez, P., Fuchs, M., Chan, H. M., Livingston, D. M. and Amati, B. (2003) MYC recruits the TIP60 histone acetyltransferase complex to chromatin. EMBO Rep. 4, 575-580.

5 Tyteca, S., Vandromme, M., Legube, G., Chevillard-Briet, M. and Trouche, D. (2006) Tip60 and p400 are both required for UV-induced apoptosis but play antagonistic roles in cell cycle progression. EMBO J. 25, 1680-1689.

6 Ikura, T., Ogryzko, V. V., Grigoriev, M., Groisman, R., Wang, J., Horikoshi, M., Scully, R., Qin, J. and Nakatani, Y. (2000) Involvement of the TIP60 histone acetylase complex in DNA repair and apoptosis. Cell. 102, 463-473.

7 Doyon, Y., Selleck, W., Lane, W. S., Tan, S. and Cote, J. (2004) Structural and functional conservation of the NuA4 histone acetyltransferase complex from yeast to humans. Mol. Cell. Biol. 24, 1884-1896.

8 Robert, F., Hardy, S., Nagy, Z., Baldeyron, C., Murr, R., Dery, U., Masson, J. Y., Papadopoulo, D., Herceg, Z. and Tora, L. (2006) The transcriptional histone acetyltransferase cofactor TRRAP associates with the MRN repair complex and plays a role in DNA doublestrand break repair. Mol. Cell. Biol. 26, 402-412.

9 Sun, Y., Jiang, X., Chen, S., Fernandes, N. and Price, B. D. (2005) A role for the Tip60 histone acetyltransferase in the acetylation and activation of ATM. Proc. Natl. Acad.

Sci. U S A. 102, 13182-13187.

10 Sun, Y., Xu, Y., Roy, K. and Price, B. D. (2007) DNA damage-induced acetylation of lysine 3016 of ATM activates ATM kinase activity. Mol. Cell. Biol. 27, 8502-8509. 
11 Puget, N., Knowlton, M. and Scully, R. (2005) Molecular analysis of sister chromatid recombination in mammalian cells. DNA Repair (Amst). 4, 149-161.

12 Legube, G., Linares, L. K., Lemercier, C., Scheffner, M., Khochbin, S. and Trouche, D. (2002) Tip60 is targeted to proteasome-mediated degradation by Mdm2 and accumulates after UV irradiation. EMBO J. 21, 1704-1712.

13 Nicolas, E., Morales, V., Magnaghi-Jaulin, L., Harel-Bellan, A., Richard-Foy, H. and Trouche, D. (2000) RbAp48 belongs to the histone deacetylase complex that associates with the retinoblastoma protein. J. Biol. Chem. 275, 9797-9804.

14 van Gent, D. C., Hoeijmakers, J. H. and Kanaar, R. (2001) Chromosomal stability and the DNA double-stranded break connection. Nat. Rev. Genet. 2, 196-206.

15 Hochegger, H., Sonoda, E. and Takeda, S. (2004) Post-replication repair in DT40

cells: translesion polymerases versus recombinases. Bioessays. 26, 151-158.

16 Sartori, A. A., Lukas, C., Coates, J., Mistrik, M., Fu, S., Bartek, J., Baer, R., Lukas, J. and Jackson, S. P. (2007) Human CtIP promotes DNA end resection. Nature. 450, 509-514.

17 Kusch, T., Florens, L., Macdonald, W. H., Swanson, S. K., Glaser, R. L., Yates, J. R., 3rd, Abmayr, S. M., Washburn, M. P. and Workman, J. L. (2004) Acetylation by Tip60 is required for selective histone variant exchange at DNA lesions. Science. 306, 2084-2087. 18 Jha, S., Shibata, E. and Dutta, A. (2008) Human Rvb1/Tip49 is required for the histone acetyltransferase activity of Tip60/NuA4 and for the downregulation of phosphorylation on H2AX after DNA damage. Mol. Cell. Biol. 28, 2690-2700.

19 Gorrini, C., Squatrito, M., Luise, C., Syed, N., Perna, D., Wark, L., Martinato, F., Sardella, D., Verrecchia, A., Bennett, S., Confalonieri, S., Cesaroni, M., Marchesi, F., Gasco, M., Scanziani, E., Capra, M., Mai, S., Nuciforo, P., Crook, T., Lough, J. and Amati, B. (2007) Tip60 is a haplo-insufficient tumour suppressor required for an oncogene-induced DNA damage response. Nature. 448, 1063-1067.

20 Eymin, B., Claverie, P., Salon, C., Leduc, C., Col, E., Brambilla, E., Khochbin, S. and Gazzeri, S. (2006) p14ARF activates a Tip60-dependent and p53-independent

ATM/ATR/CHK pathway in response to genotoxic stress. Mol. Cell. Biol. 26, 4339-4350.

21 Mirzoeva, O. K. and Petrini, J. H. (2001) DNA damage-dependent nuclear dynamics of the Mre11 complex. Mol .Cell. Biol. 21, 281-288.

22 Fuchs, M., Gerber, J., Drapkin, R., Sif, S., Ikura, T., Ogryzko, V., Lane, W. S., Nakatani, Y. and Livingston, D. M. (2001) The p400 complex is an essential E1A transformation target. Cell. 106, 297-307.

23 Li, H., Cuenin, C., Murr, R., Wang, Z. Q. and Herceg, Z. (2004) HAT cofactor Trrap regulates the mitotic checkpoint by modulation of Mad1 and Mad2 expression. Embo J. 23, 4824-4834.

24 Yang, Y. G., Saidi, A., Frappart, P. O., Min, W., Barrucand, C., Dumon-Jones, V., Michelon, J., Herceg, Z. and Wang, Z. Q. (2006) Conditional deletion of Nbs1 in murine cells reveals its role in branching repair pathways of DNA double-strand breaks. Embo J. 25, 5527-5538.

25 Yuan, Z., Zhang, X., Sengupta, N., Lane, W. S. and Seto, E. (2007) SIRT1 regulates the function of the Nijmegen breakage syndrome protein. Mol Cell. 27, 149-162.

26 Lee, J. H. and Paull, T. T. (2007) Activation and regulation of ATM kinase activity in response to DNA double-strand breaks. Oncogene. 26, 7741-7748.

27 Mattera, L., Escaffit, F., Pillaire, M. J., Selves, J., Tyteca, S., Hoffmann, J. S., Gourraud, P. A., Chevillard-Briet, M., Cazaux, C. and Trouche, D. (2009) The p400/Tip60 ratio is critical for colorectal cancer cell proliferation through DNA damage response pathways. Oncogene. 28, 1506-1517.

28 Ikura, T., Tashiro, S., Kakino, A., Shima, H., Jacob, N., Amunugama, R., Yoder, K., Izumi, S., Kuraoka, I., Tanaka, K., Kimura, H., Ikura, M., Nishikubo, S., Ito, T., Muto, A., Miyagawa, K., Takeda, S., Fishel, R., Igarashi, K. and Kamiya, K. (2007) DNA damagedependent acetylation and ubiquitination of H2AX enhances chromatin dynamics. Mol. Cell. 
Biol. 27, 7028-7040.

\section{Figure Legends}

Figure 1: Tip60 is required for repair of DSBs by homologous recombination

A) Cell survival after ionizing radiation or mitomycin C exposure. U2OS cells transfected with the indicated siRNA were subjected to increasing doses, as indicated, of irradiation (left panel) or mitomycin C (right panel) and subjected to a clonogenic assay. Results are the mean $+/-$ sd from three independent experiments. A typical western blot assaying the effects of Tip60 siRNA on the expression of Tip60 (using the anti-Tip60 LM antibody, see Exprimental Procedures) and of $\alpha$-tubulin (T6199 antibody, Sigma) as a loading control is shown in the lower panel. B) Effect of the Tip60 siRNAs in U2OS cells on homology directed repair of DSB. The mre11 and rad50 siRNAs are used as positive controls. Results represent the mean +/- sd from 3 to 4 independent experiments. Lower panel: Expression of the exogenous myctagged I-SceI endonuclease was checked by western blot. The stars (*) indicate non-specific bands.

\section{Figure 2: Tip60 is important for $\gamma \mathrm{H} 2 \mathrm{AX}$ foci formation}

Cells were electroporated with the indicated siRNA and 48h later exposed to IR ( 8 Gy) and fixed $1 \mathrm{~h}$ or $6 \mathrm{~h}$ post irradiation. Cells were then subjected to an immunofluorescence assay using an anti-phospho H2AX antibody and DAPI stained. Representative images of unirradiated cells (NT) or cells fixed 1 hour following irradiation (8 Gy) are shown in A (see supplementary data 4 for enlargement of typical cells). The quantification of a representative experiment is plotted in B. The histogram represents the mean $+/-$ sd from five different fields (at least 250 nuclei were examined). Similar results were observed in three independent experiments.

\section{Figure 3: Tip60 is involved in MRN foci formation}

Cells were electroporated with the indicated siRNA and 48h later exposed to IR ( 8 Gy) and fixed $1 \mathrm{~h}$ or $6 \mathrm{~h}$ post irradiation. Cells were then subjected to an immunofluorescence assay using an anti-Rad50 antibody and DAPI stained. Representative images of unirradiated cells (NT) or cells fixed 6 hours following irradiation (8 Gy) are shown in A (see supplementary data 4 for enlargement of typical cells). The quantification of a representative experiment shown is plotted in B. The histogram represents the mean $+/-$ sd from five different fields (at least 250 nuclei were examined). Similar results were observed in three independent experiments.

C) U2OS treated as in A) were subjected to a western blot to detect Rad50, Mre11, Nbs1 and HDAC 1, 2 and 3 (as loading controls) protein levels.

\section{Figure 4: Identification of a Tip60/MRN complex}

A) HeLa nuclear extracts $(250 \mu \mathrm{g})$ were immunoprecipitated with the indicated antibody and the presence of TRRAP, Rad50, Nbs1 and Mre11 was analyzed by western blot. B) Same as in A except that the presence of Rad50, Nbs1 and Mre11 only was analyzed by western blot.

\section{Figure 5: The Tip60/MRN complex does not contain $\mathrm{p400}$}

A) Jurkat nuclear extracts $(250 \mu \mathrm{g})$ were immunodepleted by three successive rounds of incubation with agarose beads coated with the anti-p400 or the anti-HA antibody. Depleted extracts were then immunoprecipitated with the anti-Tip60 DT or the anti-HA antibody. The presence of Rad50, Nbs1 and Mre11 in the inputs was analyzed by western blot. B) The presence of p400 in Tip60 immunoprecipitates using the extracts analysed in A was assayed 
by western blot. C) The presence of Rad50 and Nbs1 in Tip60 immunoprecipitates using the extracts analysed in A was assayed by western blot. D) Fractions obtained by size exclusion chromatography of Jurkat nuclear extracts (containing multimolecular complexes of decreasing molecular weight) were tested by western blot using anti-p400, anti-Rad50, antiNbs1, anti-Mre11 and anti-Tip60 (Tip60 LM) antibody, as indicated. E) Fractions 5, 6, 8 and 9 were immunoprecipitated with the anti-Tip60 DT antibody and immunoprecipitates were tested for the presence of $\mathrm{p} 400$ and Rad50 antibodies.

\section{Figure 6: TRRAP mediates the interaction between Tip60 and MRN proteins}

U2OS cells were transfected using the C1 or TRRAP siRNA, as indicated. 24 hours later, they were transfected by the pcDNA3-Tip60 or the empty vector, as indicated. 48 hours later, nuclear extracts were prepared and immunoprecipitated using the anti-Tip60 DT antibody, or no antibody as a control. The amounts of TRRAP, p400, Rad50 and HDAC1-3 (as a loading control) in the inputs (left panel) and the amount of p400 and Rad50 in the immunoprecipitates (right panel) were tested by western blot. Note that endogenous 400 protein expression increases upon Tip60 overexpression probably because it is stabilized. 
A

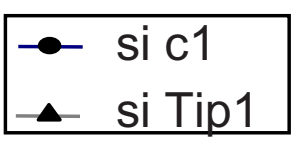

B
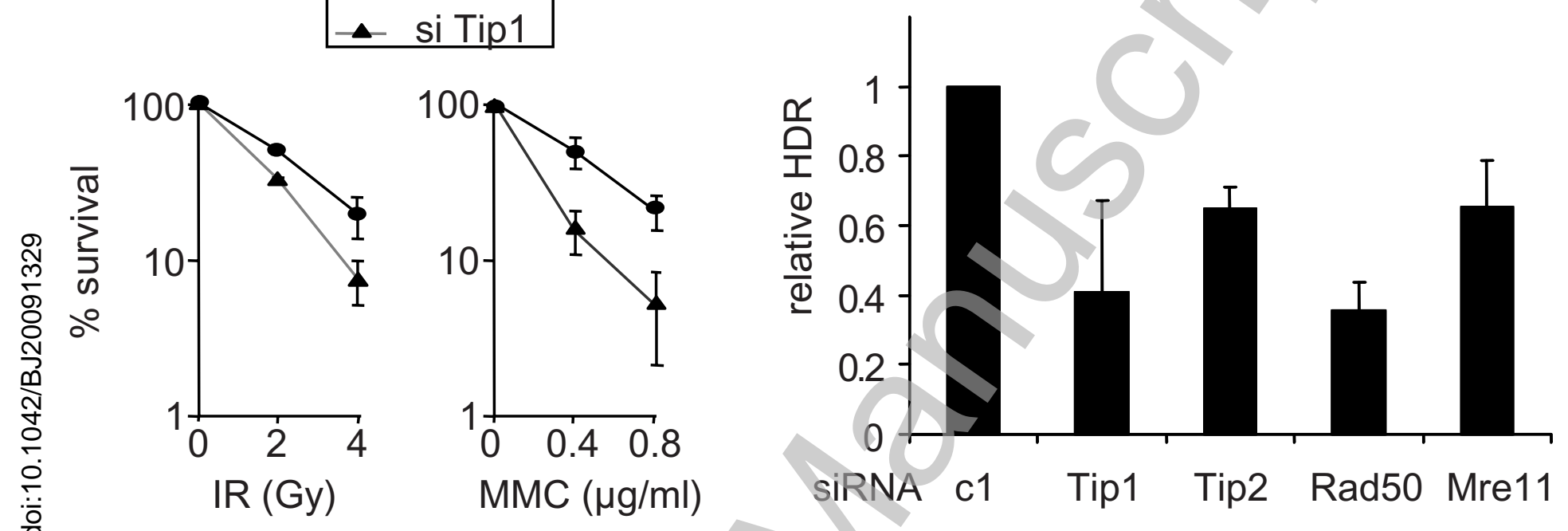

$\$$

ヘ̊

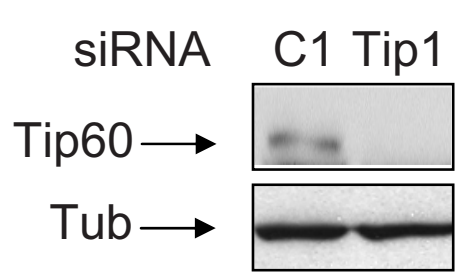

PCMV-I-Scel

siRNA c1 Tip1 c1 Tip1

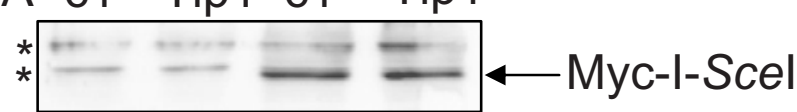

Figure 1

Chailleux et al 
A

DAPI

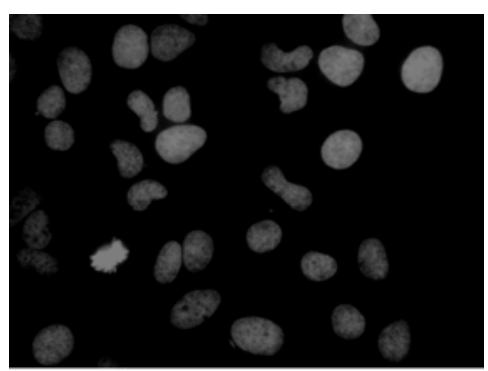

NT
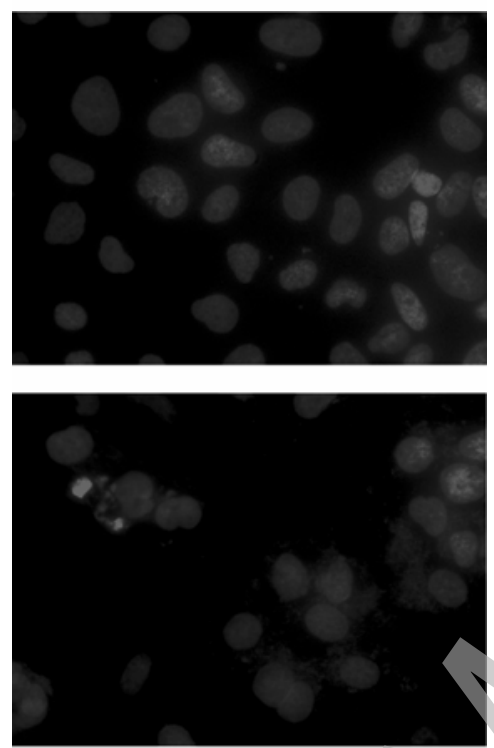

8 Gy
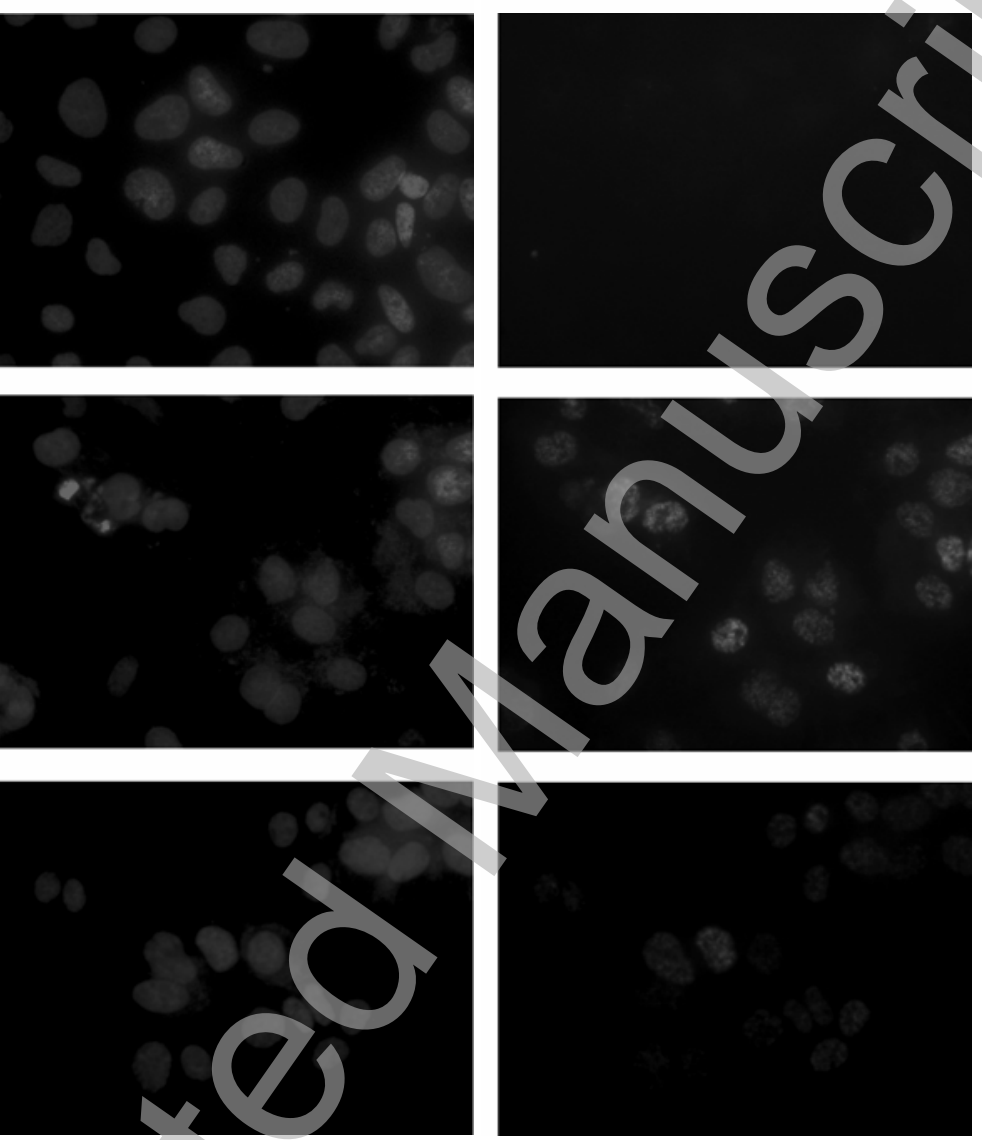

si c1

si Tip1

si Tip1

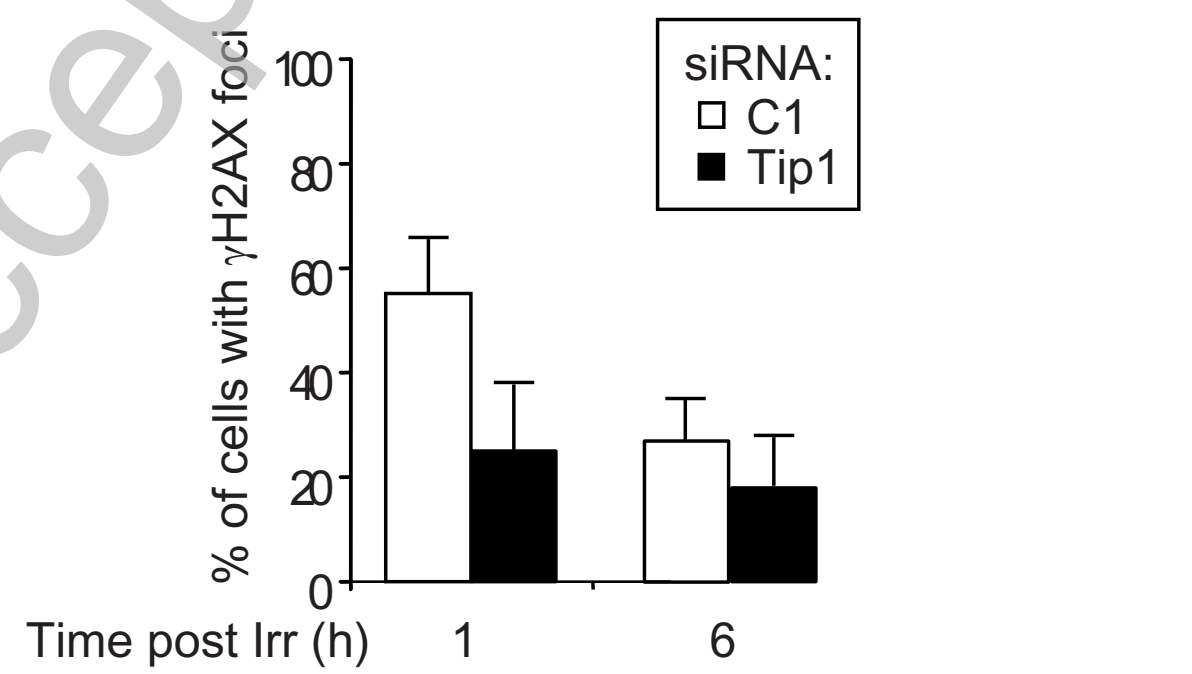

B

Figure 2

Chailleux et al

Licenced copy. Copying is not permitted, except with prior permission and as allowed by law. (c) 2010 The Authors Journal compilation (c) 2010 Portland Press Limited 


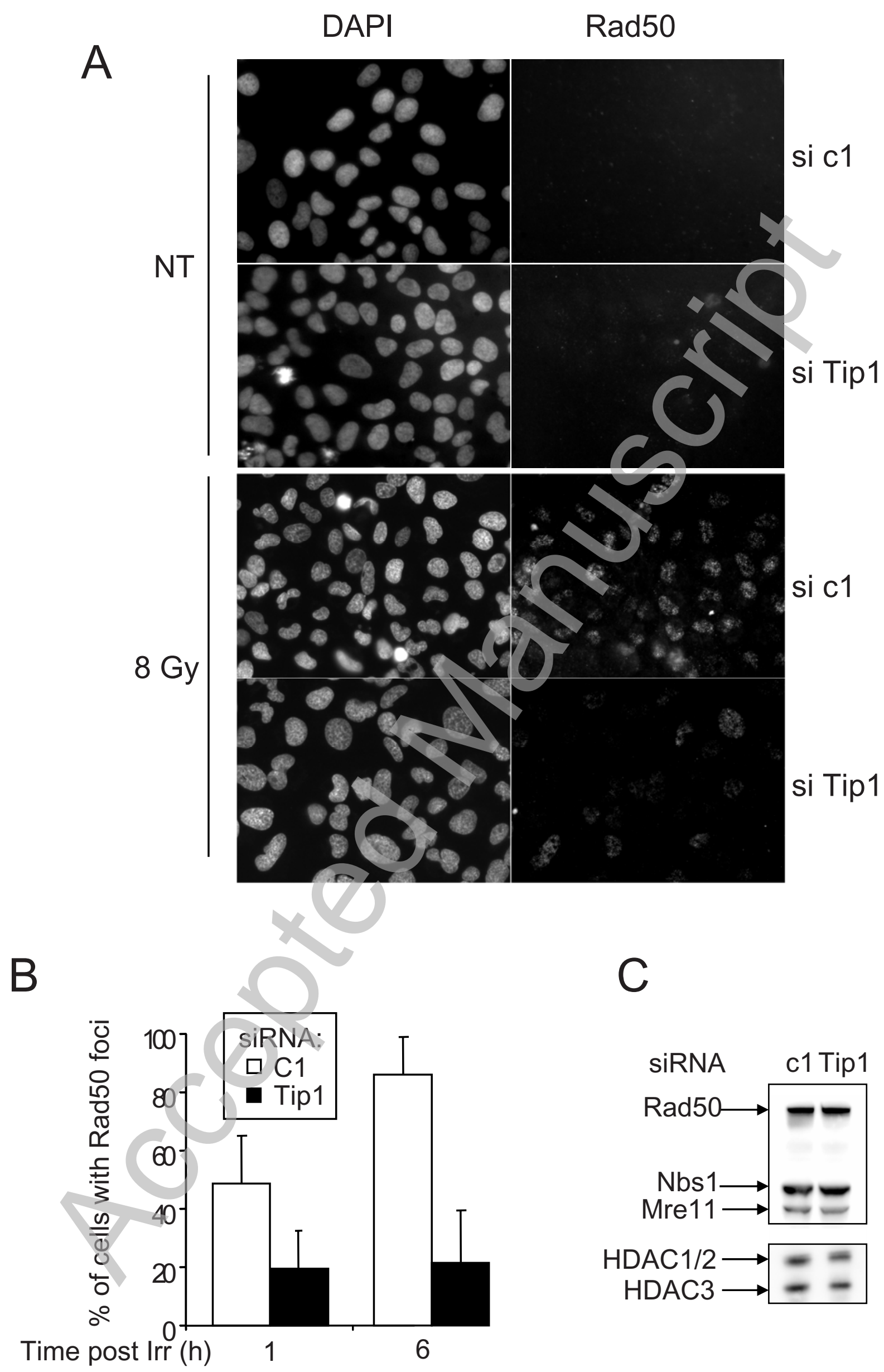

Figure 3

Chailleux et al

Licenced copy. Copying is not permitted, except with prior permission and as allowed by law. (C) 2010 The Authors Journal compilation (c) 2010 Portland Press Limited 
A

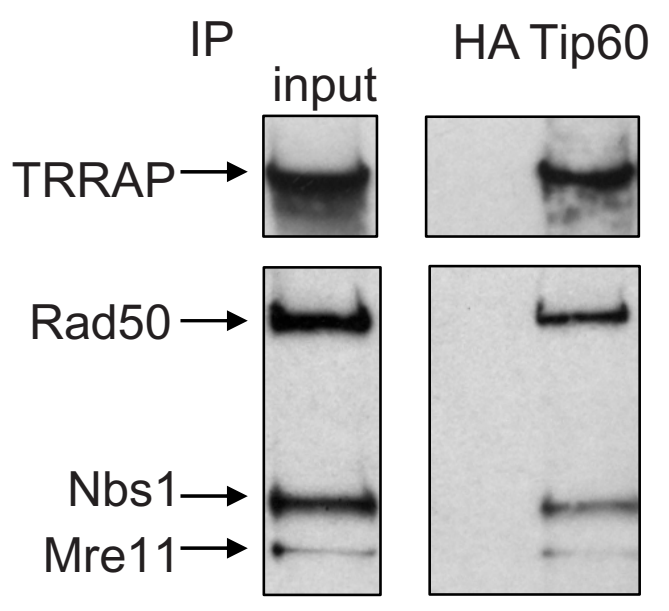

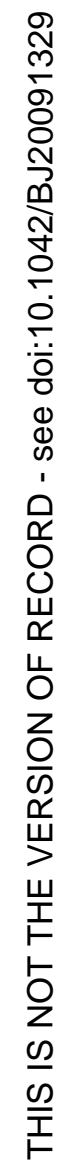

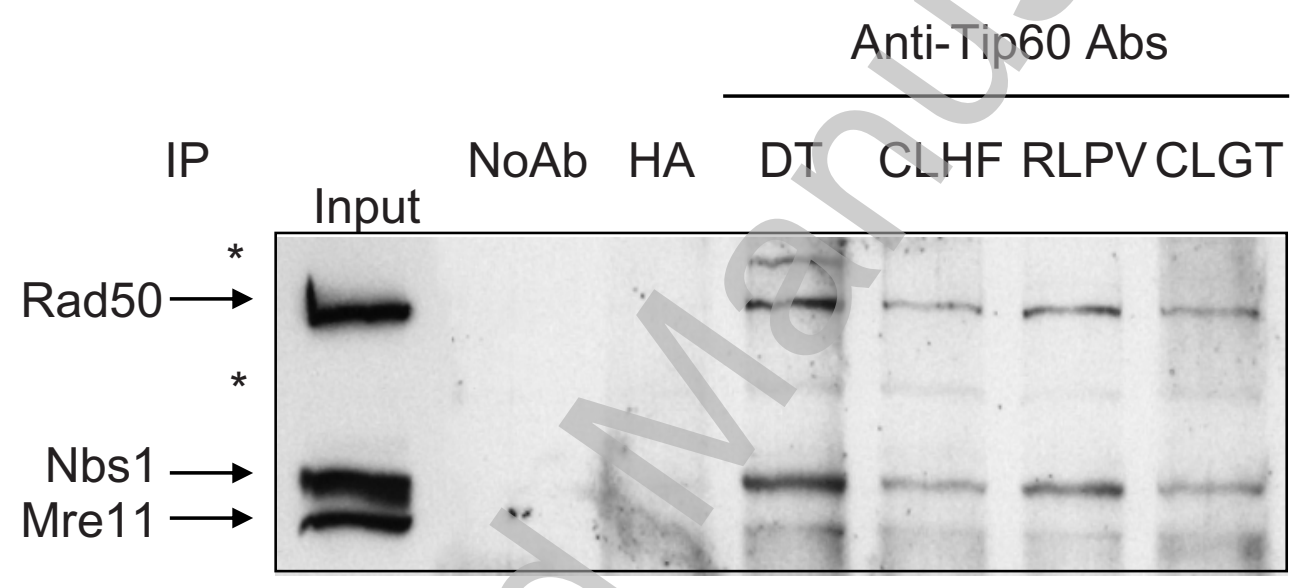

Figure 4

Chailleux et al 
Figure 5

Chailleux et al

A

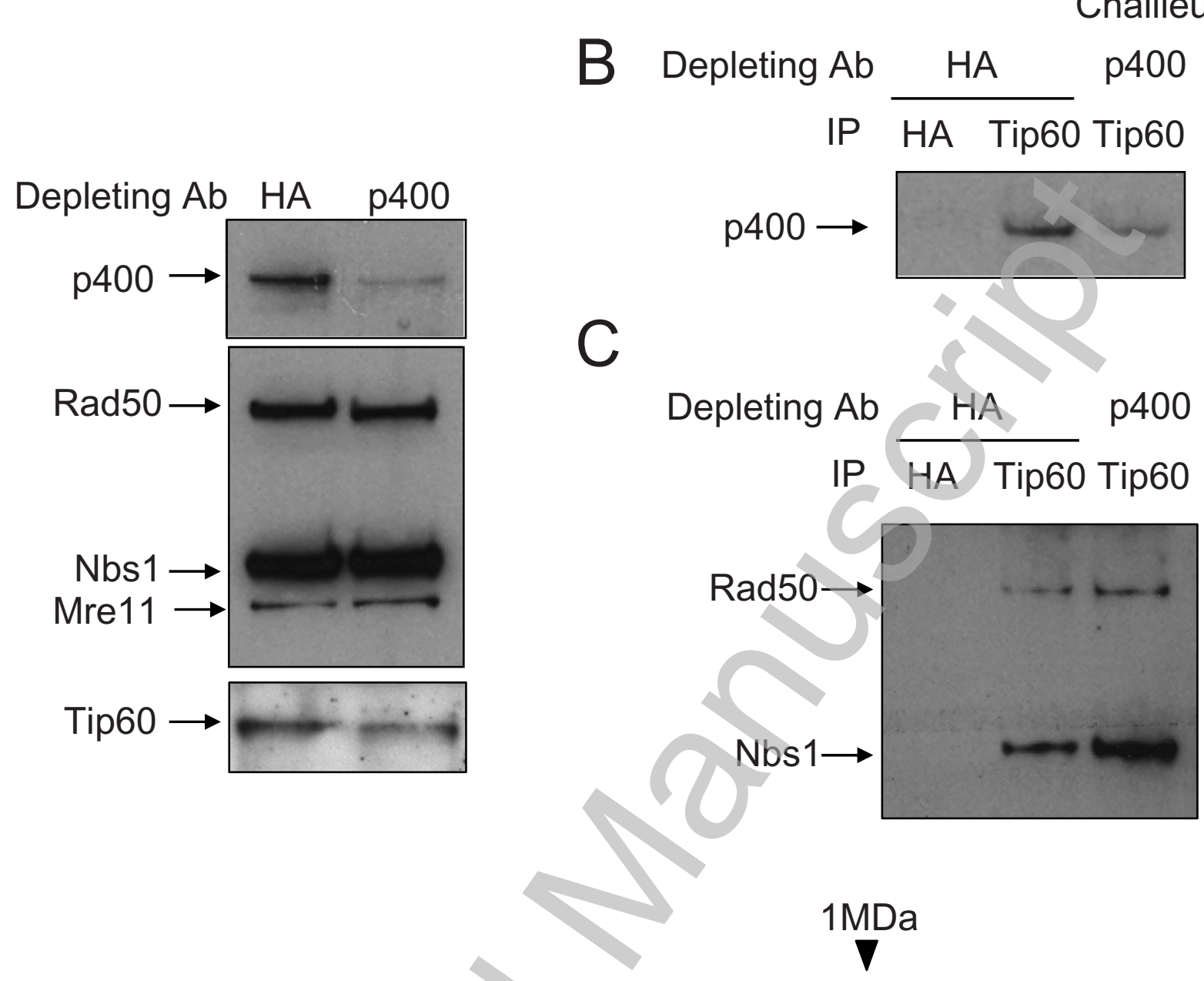

B Depleting Ab HA p400

IP HA Tip60 Tip60

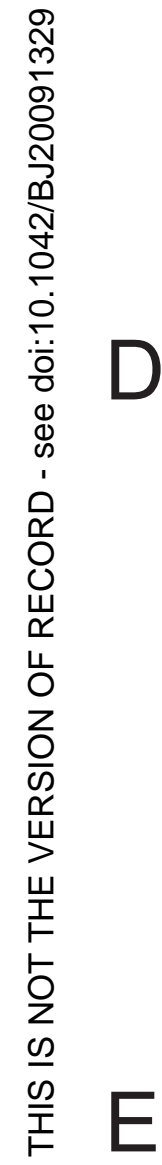
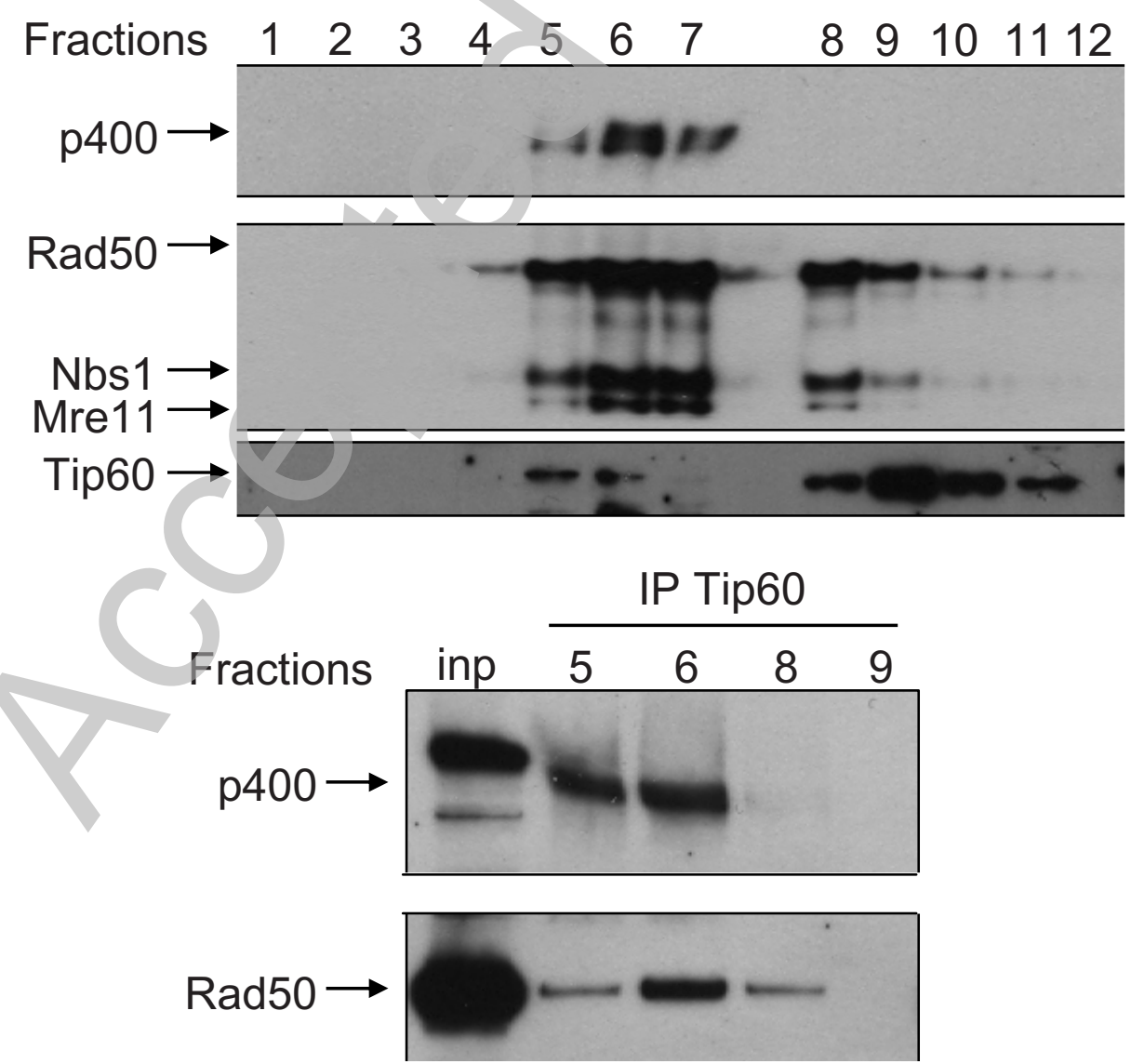

Licenced copy. Copying is not permitted, except with prior permission and as allowed by law. (C) 2010 The Authors Journal compilation (c) 2010 Portland Press Limited 
inputs

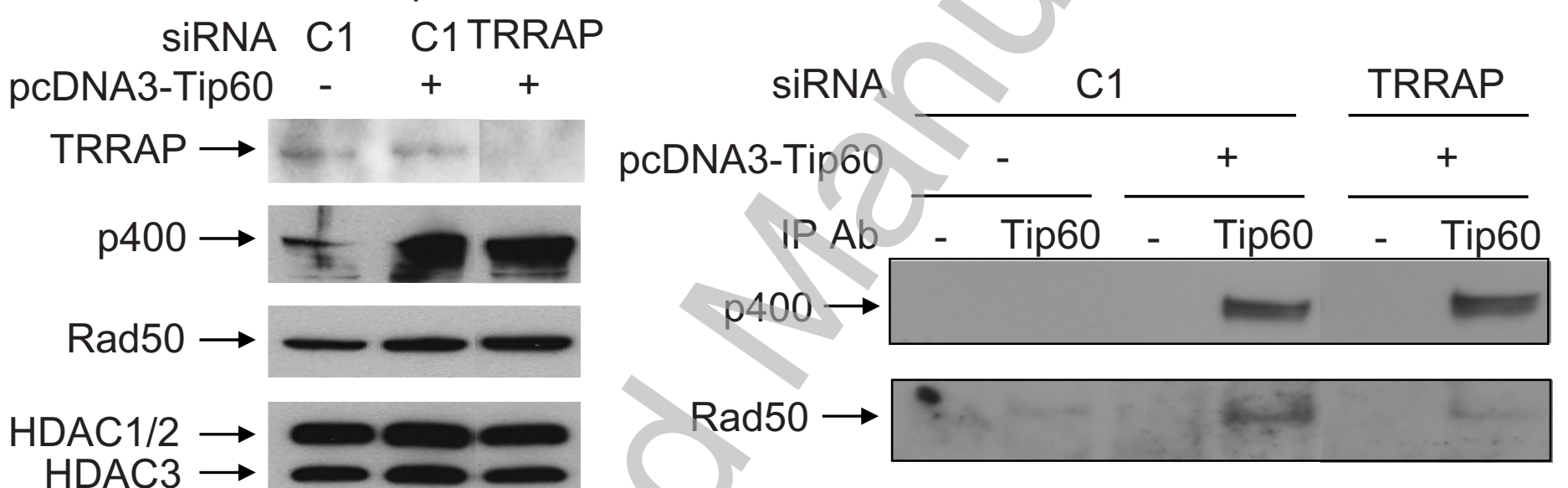

Figure 6

Chailleux et al 